\title{
Effect of Acetaminophen Against the Background of Alimentary Protein Deficiency on the Features of Sulfur-Containing Amino Acids Metabolism in Rats
}

\section{Halyna Kopylchuk* and Ivanna Nykolaichuk}

Yuriy Fedkovych Chernivtsi National University, Educational and Scientific Institute of Biology, Chemistry and Bioresourses, Lesya Ukrainka, Ukraine

*Corresponding Author: Halyna Kopylchuk, Yuriy Fedkovych Chernivtsi National University, Educational and Scientific Institute of Biology, Chemistry and

Bioresourses, Lesya Ukrainka, Ukraine.
Received: December 29, 2021

Published: January 24, 2022

(C) All rights are reserved by Halyna

Kopylchuk and Ivanna Nykolaichuk.

\section{Abstract}

In recent years, there has been extensive evidence of the involvement of disorders in the metabolism of sulfur-containing amino acids - methionine, cysteine and homocysteine - in the development of several diseases. These amino acids share common degradation pathways, and their intermediates play a role in regulating the activity of homocysteine remethylation and enzymes transsulfuration.

In this study, we aimed to evaluate the effect of acetaminophen on the background of dietary protein deficiency on the metabolism of sulfur-containing amino acids in rats: distribution of sulfur-containing amino acids - homocysteine, cysteine and methionine - in blood serum and hepatocytes, activity of key enzymes of the transsulfuration pathway of homocysteine metabolism - cystathionine beta-synthase, cystathionine gamma-lyase.

During the experiment, the experimental animals consumed a semi-synthetic diet AIN-93 in accordance with the recommendations of the American Institute of Nutrition. In order to model the alimentary protein deprivation rats received a low-protein diet daily for 28 days, which contained $1 / 3$ of the generally accepted daily requirement of protein. After four weeks of keeping animals on an experimental diet, acute toxic damage with acetaminophen was modelled. The toxin was administered at $1250 \mathrm{mg} / \mathrm{kg}$ of animal weight as a suspension in a $2 \%$ solution of starch gel once a day for 2 days.

Our results indicate that the conditions of alimentary protein deprivation and acetaminophen toxic injury in hepatocytes and blood serum of animals disrupts the distribution of sulfur-containing amino acids (decrease in methionine, increase in cysteine concentration) with the development of hyperhomocysteinemia: in the absence of dietary protein - a mild form, with the introduction of toxic doses of acetaminophen - moderate.

The results confirm the causal relationship between the functioning of the transsulfuration pathway of homocysteine (decreased cystathionine $\beta$-synthase activity with simultaneous activation of cystathionine $\gamma$-lyase in animal hepatocytes) and the maximum increase in the level of this amino acid in blood serum under conditions of acetaminophen-toxic injury.

Elevated blood homocysteine levels can therefore be considered as a prognostic marker of functional abnormalities in the liver transsulfuration pathway and can be used in the diagnosis of hepatic pathologies.

Keywords: Amino Acids; Sulfur; Methionine; Cysteine; Homocysteine; Metabolism; Transsulfuration; Cystathionine Beta-Synthase; Cystathionine Gamma-Lyase; Acetaminophen; Alimentary Protein Deficiency; Liver; Rats 


\section{Introduction}

Recent decades have been characterized by a rapid increase in homeostatic organ pathologies developing against the background of nutritional imbalances in dietary intake [1,2]. Nutritional protein deficiency is usually accompanied by the development of protein-energy deficiency in the body and leads to depletion of the intracellular amino acid pool $[3,4]$.

Quite often, the course of alimentary-dependent diseases is intensified by the use of a wide range of drugs. The most common analgesics-antipyretics nowadays invariably include acetaminophen (N-acetyl-p-aminophenol, paracetamol) [5,6]. The increased frequency of use in recent years has raised concerns about medication-associated lesions of the body $[7,8]$.

Although acetaminophen-induced lesions have emerged as the leading cause of acute liver failure $[9,10]$, acetaminophen is now considered the main antipyretic and anti-inflammatory drug in COVID-19 treatment protocols [11-13].

At the same time, in scientific studies, acetaminophen is used as a model toxin to elucidate the mechanisms of hepatotoxicity $[14,15]$.

Acetaminophen is almost completely metabolized in liver: a small amount (2-9\%) is excreted unchanged, the rest is subject to glucuronidation ( $40-65 \%$ ) and sulfation (25 - 35\%), 5-12\% of the drug is biotransformed by cytochrome P-450 system. (CYP 450) to form the highly toxic reactive metabolite $\mathrm{N}$-acetyl-p-benzoquinonimine (NAPQI) [16,14].

In recent years, there has been extensive evidence of the involvement of disorders in the metabolism of sulfur-containing amino acids - methionine, cysteine and homocysteine (Hcy) - in the development of several diseases $[18,19]$. These amino acids share common degradation pathways, and their intermediates play a role in regulating the activity enzymes of homocysteine methylation and transsulfuration [20].

Remethylation of Hcy in liver occurs during reactions of the methionine cycle, which is completed by methionine regeneration through the use of 5-methyltetrahydrofolate or betaine, which act as donors of the methyl group. Another metabolic pathway occurs through transsulfuration, where cysteine is formed from Hcy, which is involved in the synthesis of protein, taurine, hydrogen sulfate, and glutathione [21]. The transsulfuration pathway plays a significant role in eliminating potentially dangerous excess homocysteine and, according to some data, has a significant effect on its catabolism in the blood plasma (it changes almost 78\%), whereas the conversion of Hcy to methionine provides utilization of only $2 \%$ of this compound [22].

Although the role of homocysteine in biochemical processes has been discussed for quite a long time, the main publications on the association of increased HC content with pathological conditions have appeared in recent years [20,23,24]. It is known that an increase in the content of Hcy in the blood causes an imbalance between its production and utilization pathways. At the same time, homocysteine level is considered as a pathogenic factor that can lead to the development of hyperhomocysteinemia, which is confirmed by the results of previous studies in dietary protein deficiency [25].

To evaluate the effect of acetaminophen against the background of alimentary protein deficiency on the metabolism of sulfur-containing amino acids in rats, we studied: distribution of sulfur-containing amino acids - homocysteine, cysteine and methionine - in blood serum and hepatocytes, activity of key enzymes of the transsulfuration pathway of homocysteine metabolism - cystathionine beta-synthase, cystathionine gamma-lyase.

\section{Results}

The results of our studies have shown that protein deficiency in the diet of the research groups of LPD and LPD+TI animals led to a significant decrease in methionine content (by 4.7 and 2.3 times as compared to the control) and cysteine (by 3 and 2 times respectively in comparison with the control) in hepatocytes (Table 1). At the same time, in the group of animals with toxic liver damage (TI) kept on a complete diet, there is a decrease in cysteine level (3 times compared to the control), while the amount of methionine remains at the control level.

An increased level of cysteine by 1.4-1.8 times compared to the control was registered in the blood serum of all experimental groups of animals (Table 2). At the same time, the direction of changes in methionine content is similar to its changes in hepatocytes and is determined by the provision of the diet with protein 


\begin{tabular}{|l|c|c|c|c|}
\hline \multirow{2}{*}{ Index } & \multicolumn{4}{|c|}{ Groups of animals } \\
\cline { 2 - 5 } & C & LPD & TI & LPD+TI \\
\hline Methionine, $\mu \mathrm{mol} / \mathrm{mg}$ tissue & $12.50( \pm 1.08)$ & $2.68( \pm 0.23)^{* *}$ & $13.29( \pm 1.12)$ & $5.36( \pm 0.45)^{* *}$ \\
\hline $\begin{array}{l}\text { Cysteine, } \\
\mu \mathrm{mol} / \mathrm{mg} \text { tissue }\end{array}$ & $22.22( \pm 2.12)$ & $7.41( \pm 0.65)^{* *}$ & $7.41( \pm 0.53)^{* *}$ & $11.12( \pm 0,96)^{* *}$ \\
\hline
\end{tabular}

Table 1: Content of sulfur-containing amino acids in rat hepatocytes during alimentary protein deprivation and acetaminophen toxic injury $(\mathrm{M} \pm \mathrm{m}, \mathrm{n}=40)$.

Note (hereafter):

C - Animals Receiving a Complete Semi-Synthetic Diet (control).

LPD - Animals on A Low-Protein Diet.

TI - Animals Simulated by Acetaminophen Toxicity.

LPD+TI - Animals that were Simulated with Toxic Injury on Low-Protein Diet.

** - All values are significant $\mathrm{p}<0.0$.

(decreasing in the LPD and LPD+TI groups) with preservation of the control level in the TI group (Table 2).

\begin{tabular}{|l|c|c|c|c|}
\hline \multirow{2}{*}{ Index } & \multicolumn{4}{|c|}{ Groups of animals } \\
\cline { 2 - 5 } & C & LPD & TI & LPD+TI \\
\hline Methionine, $\mu \mathrm{mol} / \mathrm{L}$ & $5.54( \pm 0.48)$ & $3.58( \pm 0,32)^{* *}$ & $6.25( \pm 0,61)$ & $4.46( \pm 0.40)^{* *}$ \\
\hline $\begin{array}{l}\text { Cysteine, } \\
\mu \mathrm{mol} / \mathrm{L}\end{array}$ & $8.15( \pm 0,75)$ & $11.11( \pm 1,02)^{* *}$ & $18.52( \pm 0,43)^{* *}$ & $14.81( \pm 0,860)^{* *}$ \\
\hline
\end{tabular}

Table 2: Sulfur-containing amino acid content in serum of rats during alimentary protein deprivation and acetaminophen toxic injury $(\mathrm{M} \pm \mathrm{m}, \mathrm{n}=40)$.

Note: ${ }^{* *}$ - All values are significant $\mathrm{p}<0.01$.

As for the homocysteine content in the blood serum of rats that were on a low-protein diet (LPD), this index increased 2-fold compared with the values of the control group of animals, reaching a level of $28.2( \pm 1.94) \mu \mathrm{mol} / \mathrm{L}$, which indicates the development of mild hyperhomocysteinemia (Figure 1).

In the TI and LPD+TI rat groups, the serum Hcy content was $51.5( \pm 4.97) \mu \mathrm{mol} / \mathrm{L}$ and $52.9( \pm 5.050) \mu \mathrm{mol} / \mathrm{L}$, respectively, which in turn indicated the development of a moderate form of hyperhomocysteinemia.

The study of the activities of the main enzymes transsulfuration pathway of homocysteine in rat hepatocytes revealed a statistically probable decrease in cystathionine $\beta$-synthase activity in all experimental groups of animals (Figure $2 \mathrm{a}$ ). The maximum decrease

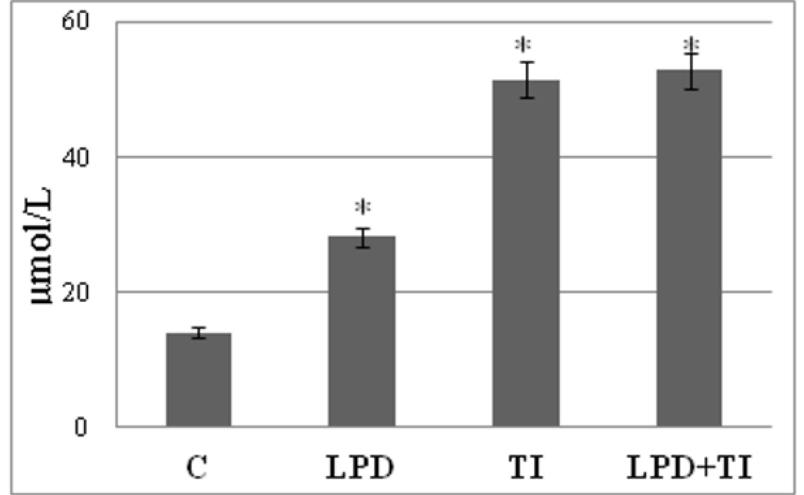

Figure 1: Homocysteine content in serum of rats upon alimentary protein deprivation and acetaminophen toxic injury Note: * all values are significant $\mathrm{p}<0.05$ 
in the index of the studied enzymatic activity (4-fold compared to the control) was observed in the groups of rats given toxic doses of acetaminophen, regardless of the protein feeding regime.

At the same time, we found an increase in the activity of cystathionine $\gamma$-lyase in the hepatocytes of all experimental groups of rats with maximum values when acetaminophen toxic doses were administered against the background of protein deficiency (3.5fold as compared to the control) (Figure 2b).
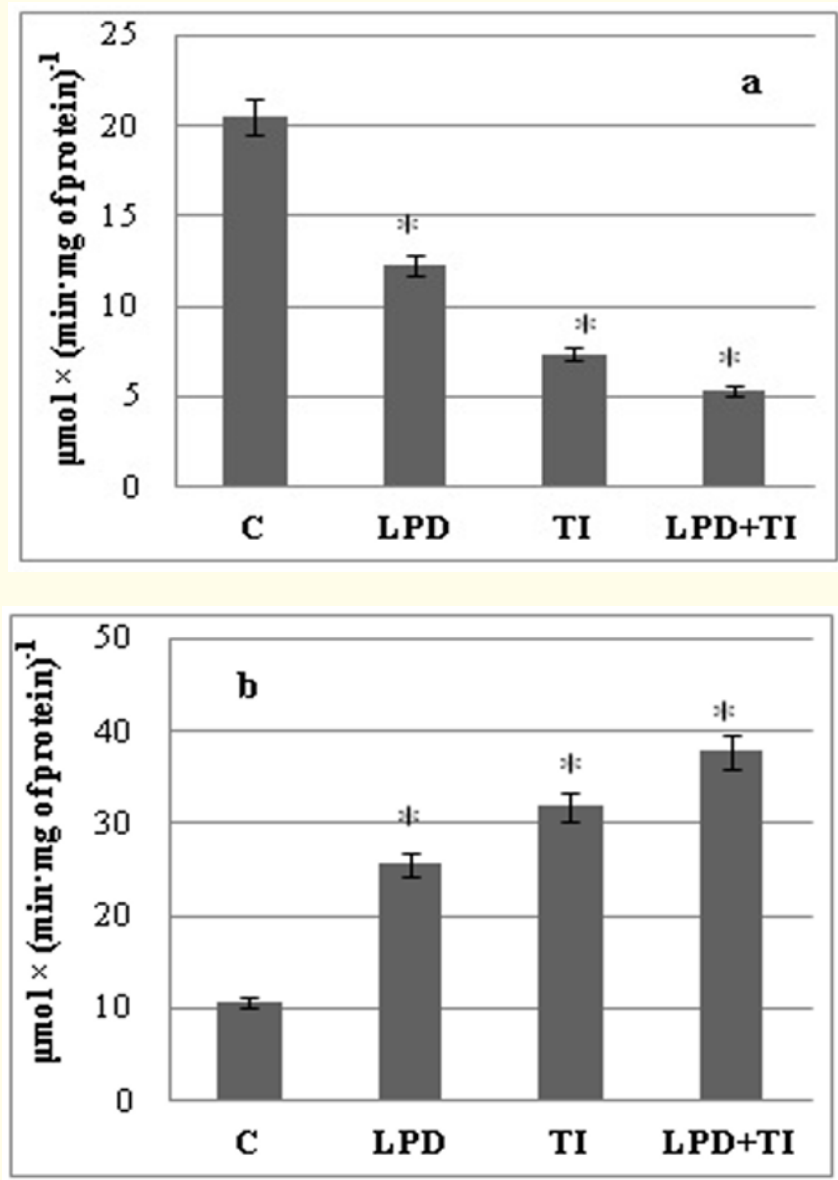

Figure 2: Activity of cystathionine $\beta$-synthase (a) and cystathionine $\gamma$-lyase (b) under conditions of alimentary protein deprivation and acetaminophen toxicity Note: ${ }^{*}$ - all values are significant $\mathrm{p}<0.05$.

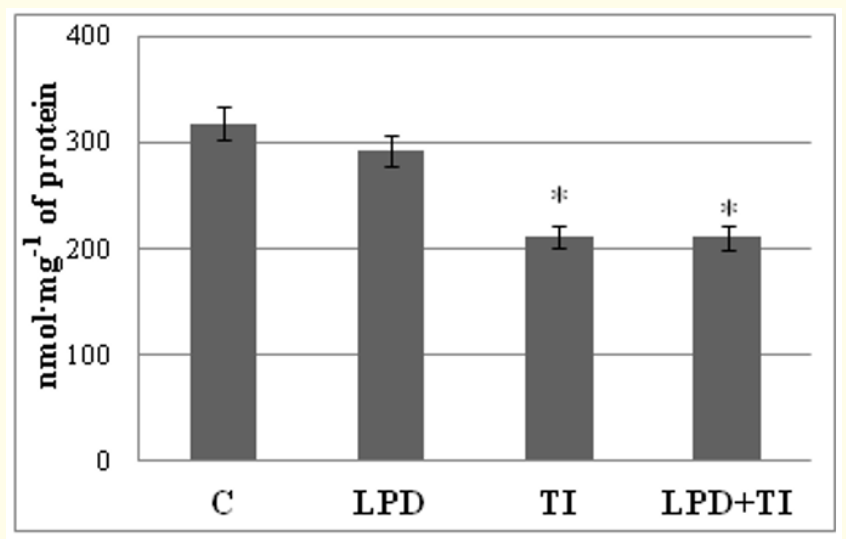

Figure 3: Content of sulfhydryl groups in rat hepatocytes under conditions of alimentary protein deprivation and acetaminophen toxic injury

Note: * all values are significant $\mathrm{p}<0.05$.

A study of the content of protein sulfhydryl groups in rat hepatocytes during administration of acetaminophen and alimentary protein deficiency (Figure 3) showed a probable decrease in the indices as compared with the control in the groups of animals receiving toxic doses of acetaminophen regardless of the amount of protein in the diet.

\section{Discussion}

Sulfur-containing amino acids have common degradation pathways, and their intermediate metabolites act as regulators of homocysteine transsulfuration and remethylation enzyme activities [20].

Homocysteine is produced in the metabolic cycle of the essential amino acid methionine by its remethylation. Methionine is the only source of homocysteine in the body. Homocysteine content in food is very low, and it is the methionine and homocysteine requirements that are provided by food methionine [22].

Here, the analysis of the results obtained confirms the key and decisive role of protein deficiency in the impairment of methionine metabolism. Noting that an alternative pathway for homocysteine remethylation and methionine formation is the vitamin $\mathrm{B}_{12}$ independent transfer of the betaine methyl group to homocysteine involving betaine-homocysteine S-methyltransferase [26], we 
hypothesize that it is this homocysteine conversion pathway that maintains methionine content at a stable level when toxic doses of acetaminophen are administered.

At the same time, homocysteine emerges as an intermediate in the synthesis of cysteine from methionine by transsulfuration. Therefore, it is understandable that a decrease in methionine content in liver cells of experimental groups of animals leads to a decrease in cysteine concentration.

Cysteine is a limiting link in glutathione synthesis [27]; it can be assumed that a decrease in the concentration of this amino acid would be paralleled by a decrease in the products of its synthesis. However, the results of the studies described in the paper [28] indicate a low relationship between cysteine levels and the products of its synthesis, which suggests several regulators of glutathione content. Glutathione is a reserve of cysteine in tissues and is regarded as a reserve and transport form of this amino acid in the body. It should be noted that the decreased level of cysteine in rat hepatocytes during protein deficiency and acute toxic injury is evidenced by the decreased content of reduced glutathione in liver cells.

The three-fold decrease of cysteine content in hepatocytes of animals with acetaminophen-induced injury has been shown in previous works to be accompanied by the development of oxidative stress, which is probably associated with increased utilization of sulfhydryl groups of cysteine for glutathione synthesis.

Therefore, the deficiency of methionine and cysteine in liver cells of protein-energy-deficient rats that we have found may be a consequence of both their alimentary deficiency and impaired synthesis.

It is known that the concentration of free amino acids in biological fluids and tissues is an integral indicator of homeostasis that characterises protein metabolism, and the patterns of amino acid pool formation in the body objectively reflect the state of metabolic balance.

Table 2 shows that both protein deficiency and the administration of toxic doses of acetaminophen have a unidirectional effect towards an increase in serum cysteine. One might assume that the increase in serum cysteine concentration is related to the fact, described in the literature, that there is a competitive relationship between individual sulphur-containing amino acids in transport systems. Cysteine takes part in I and II transport systems almost along with other sulphur-containing amino acids. Probably as part of the body's ensuring requirements, other amino acids, competing with cysteine for the transport protein, penetrate the biological cell membranes more easily than cysteine, which results in a higher serum content.

By analogy with the pathogenesis of hyperhomocysteinemia, the mechanism of adverse effects of excess cysteine may also be related to the covalent modification (cysteination) of proteins with consequent changes in their functional activity [29].

There is also a view expressed in the literature that there is an antagonistic relationship between homocysteine and cysteine contents. It has been noted that in the case of excess cysteine, homocysteine is displaced from mixed disulphides (protein-homocysteine type), and, instead, cysteine is incorporated.

Considering that the transport of cysteine through the ASC system [30] under hyperhomocysteinemia may work mainly on the release of this amino acid from the cell, an increase of its concentration in blood serum appears logical. In the blood, cysteine is mainly in an oxidised state, binding to proteins to form cystine and mixed disulphides. Cystine is transported into cells by system $\mathrm{Xc}^{-}$ while exchanging for glutamate, which is also required for glutathione synthesis. Thus, it can be expected that GSH homeostasis in this case would be ensured by the regulation of cysteine transport between cells and the extracellular environment.

Homocysteine in blood plasma is known to occur predominantly in three forms: free homocysteine (1\%), homocysteine disulfide with cysteine (homocysteine) (20 - 30\%) and bound to protein, in particular to albumin (70-80\%) [31]. The literature suggests that there is competition between homocysteine and cysteine for binding to blood albumin. It has been noted that in excess of cysteine, homocysteine is displaced from the albumin-homocysteine complex and cysteine is incorporated instead. Thus, homocysteine levels in the blood increase, and the hypercysteinemia that we have identified may be an additional pathogenetic factor in hyperhomocysteinemia.

In the literature, suggestions have been made that there is competition between homocysteine and cysteine for binding to blood 
albumin. It has been noted that with excess cysteine, homocysteine is displaced from the albumin-homocysteine complex and instead, cysteine is incorporated. Thus, homocysteine levels in the blood increase, and the hypercysteinemia that we have identified may be an additional pathogenetic factor in hyperhomocysteinemia.

Since the kidneys are the main organ of homocysteine catabolism, capable of utilising up to $20 \%$ of plasma homocysteine during the passage of arterial blood through them [20], signs of acute interstitial nephritis and renal papillary necrosis identified in our laboratory can be considered another factor of plasma homocysteine accumulation, whereby the filtering capacity of homocysteine by the kidneys will be significantly reduced.

Studies [32] have shown that hyperhomocysteinemia is a metabolic disorder associated with the progression of hepatic fibrosis. The profibrogenic effect of hyperhomocysteinemia can occur both through the direct effects of homocysteine and through the metabolic disorders associated with hyperhomocysteinemia. It is shown that homocysteine initiates the expression of tissue inhibitor of metalloproteinase- 1 in co-culture of hepatocytes and hepatic stellate cells and plays the role of hepatic fibroblast promoter. Mediated mechanisms of the profibrogenic effects of excess homocysteine are reduction of vasodilator production in the liver, inhibition of methylation, initiation of oxidative stress, and development of steatosis.

The main way to eliminate a potentially dangerous excess of homocysteine in hyperhomocysteinemia is its transsulfuration with the formation of cysteine. In this process homocysteine condenses with serine to form cystathionine in a reaction catalysed by cystathionine $\beta$-synthase and under physiological conditions virtually irreversible. Cystathionine is further degraded by cystathionine $\gamma$-lyase to form cysteine, ammonia and $\alpha$-ketobutyrate. Excess cysteine is oxidised to taurine and inorganic sulphate [33].

We found that the maximum decrease in the index of the studied enzymatic activity was observed in the groups of rats which were administered toxic doses of acetaminophen regardless of the protein nutrition regime. Noteworthy is the fact that in parallel with a decrease in cystathionine $\beta$-synthase activity in animals of the experimental groups, a state of moderate hyperhomocysteinemia develops.
On the other hand, one can assume that already at the first stage of homocysteine utilization under these conditions, the mechanisms of homocysteine transsulfuration are disrupted, an excess of this amino acid in the liver will lead to accumulation of S-adenosyl homocysteine, a potent inhibitor of methyltransferases in cells and, as a result, disruption of methylation processes, which acts as a key pathogenesis of hyperhomocysteinemia. It is known that the accumulation of homocysteine in cells automatically increases the concentration of S-adenosylhomocysteine, and a clear relationship is established between the levels of both compounds [34].

Given the data in table 1 regarding methionine content in hepatocytes, one possible mechanism for the inhibition of cystathionine $\beta$-synthase activity may be low levels of S-adenosylmethionine, an allosteric activator of cystathionine $\beta$-synthase [26].

The decrease in cystathionine $\beta$-synthase activity is obviously accompanied by a decrease in cystathionine, a substrate for the next enzyme of the transsulfuration pathway, cystathionine $\gamma$-lyase. Therefore, the seemingly paradoxical fact of increased cystathionine $\gamma$-lyase activity (Figure $2 \mathrm{~b}$ ) that we have established can be explained by the activation of an alternative condensation reaction of 2 homocysteine molecules to form homocysteinlactone and hydrogen sulfide [35].

Due to the production of hydrogen sulfide, cystathionine $\gamma$-lyase functions as a sulfhydrase of cysteine-containing proteins, regulating their functions by sulfhydrogenation through the conversion of SH-groups to SSH- groups at specific cysteine residues [36]. Most likely, the data obtained testify to the modification of SH-groups which may be accompanied by changes in the conformational structure of protein molecules. Moreover, changes in the content of SH groups in proteins reflect the causal relationship between thiol metabolism disorder and the development of hyperhomocysteinemia.

\section{Materials and Methods}

Animals

White outbred rats aged 2.5 - 3 months and weighing $130-150 \mathrm{~g}$ were used for the study. All animal procedures were performed according to international recommendations of the European Convention for the Protection of Vertebrate Animals Used for Experimental and Other Scientific Purposes (Strasburg, 1986), «General Ethical Principles for Animal Experiments», approved by the First National Congress on Bioethics (Kyiv, Ukraine, 2001). 
During the experiment, animals consumed a semi-synthetic diet AIN-93 in accordance with the recommendations of the American Institute of Nutrition on the principle of pair-feeding [37].

Acute toxic liver injury was simulated by administering acetaminophen per os to experimental animals at the rate of 1250 $\mathrm{mg} / \mathrm{kg}\left(0.5 \mathrm{LD}_{50}\right)$ of animal weight in the form of suspension in $2 \%$ starch gel solution once a day for 2 days [3].

The research model involved dividing the animals into groups

- Animals kept on a semi-synthetic diet balanced for all nutrients - control group (C).

- Animals kept on a semi-synthetic low-protein diet for 4 weeks prior to the experiment $(1 / 3$ of the normal daily protein requirements) (LPD).

- Animals that received acute acetaminophen-induced toxic injury (TI) after being fed a complete semi-synthetic diet

- Animals with acute toxic injury modeled against the background of alimentary protein deficiency (LPD+TI).

Cervical dislocation of the studied animals under light ether anesthesia was performed on days 28 and 31 of the experiment.

\section{Isolation of hepatocytes}

Hepatocytes were isolated using a non-enzymatic method. The liver tissues were perfused with Hanks' solution $\left(37^{\circ} \mathrm{C}\right)$ followed by the addition of $2 \mathrm{mM}$ EDTA to attenuate intercellular contacts [38]. Hepatocytes were obtained by low-speed centrifugation (80 g), then resuspended in Hanks' solution without EDTA and counted in a Goryaev chamber under a Spencer microscope (USA) after staining with $0.2 \%$ trypan blue solution. Hepatocyte viability was $94 \pm 2 \%$.

\section{Chromatography}

Determination of methionine and cysteine content in proteinfree blood serum and hepatocyte samples was performed using a T-339 automatic amino acid analyzer from Microtechna (Czech Republic).

\section{Enzyme-linked immunosorbent assay (ELISA)}

Homocysteine concentration in blood plasma was determined by enzyme immunoassay using Axis-Shield kit (UK).

\section{Enzymatic activity}

The activity of cystathionine $\gamma$-lyase was assessed by the amount of formed hydrogen sulfide. The reaction medium contained 0.67 $\mathrm{mM}$ pyridoxal phosphate, $3.3 \mathrm{mM}$ L-cysteine, $0.083 \mathrm{M}$ tris- $\mathrm{HCl}$ buffer, $\mathrm{pH}$ 8.5. The obtained samples were incubated at $37^{\circ} \mathrm{C}$ in tubes sealed with foil (to avoid loss of $\mathrm{H}_{2} \mathrm{~S}$ ). The reaction was stopped by cooling the tubes on ice, and a $1 \%$ solution of zinc acetate, $20 \mathrm{mM}$ $\mathrm{N}, \mathrm{N}$-dimethyl paraphenyldiamine in $7.2 \mathrm{M} \mathrm{HCl}$, and $30 \mathrm{mM} \mathrm{FeCl}_{3}$ in $1.2 \mathrm{M} \mathrm{HCl}$ was added. After deproteinization, the extinction of the supernatant was measured on a CARY 60 spectrophotometer (USA) at $670 \mathrm{~nm}$.

Cystathionine $\beta$-synthase activity was calculated as the difference between the total activity (cystathionine $\gamma$-lyase + cystathionine $\beta$-synthase) and cystathionine $\gamma$-lyase activity. To determine total enzyme activity, we used medium that contained $0.67 \mathrm{mM}$ pyridoxal phosphate, $3.3 \mathrm{mM}$ L-cysteine, $0.083 \mathrm{M}$ tris- $\mathrm{HCl}$ buffer, pH 8.5-, and 3.3-mM homocysteine at final concentrations [39].

\section{Determination of the content of thiol groups in proteins}

The content of thiol groups in proteins (SH-groups) was determined by the method based on the use of 5,5'-dithio-bis-2-nitrobenzoic acid (DTNBA), which reacts with SH-groups, forming a mixed disulfide and releasing a thionitrophenyl anion, whose amount is directly proportional to the number of free $\mathrm{SH}$-groups in the proteins reacted with DTNBA [40].

The protein content in the experimental samples was determined by the Bradford method.

\section{Statistical data analysis}

Statistical analysis of the data was performed using Microsoft Office Excel 2016 (activation key JXTBB-4NX7D-B2PBT-32HKFWFG9) and STATISTICA 6.0. The values obtained in the groups of experimental animals were compared with the control using Student's t-test and non-parametric Mann-Whitney $U$ test. For this purpose we calculated the arithmetic mean of independent determinations and standard deviations. The data were considered significant when $\mathrm{p} \leq 0.05(*), \mathrm{p} \leq 0.01\left({ }^{* *}\right)$.

\section{Conclusions}

Thus, in alimentary protein deprivation and acetaminophen toxic injury, the distribution of sulphur-containing amino acids in 
hepatocytes and serum of animals is distorted with the development of hyperhomocysteinemia. At that, the most significant disturbance of transsulfuration pathway of homocysteine metabolism, characterised by decrease of cystathionine $\beta$-synthase activity with simultaneous activation of cystathionine $\gamma$-lyase in animal hepatocytes, occurs with administration of toxic doses of acetaminophen. Elevated blood homocysteine levels can therefore be considered as a prognostic marker of functional abnormalities in the liver transsulfuration pathway and can be used in the diagnosis of hepatic pathologies.

The data we obtained bring out the key points in understanding the metabolic processes of methionine, homocysteine and cysteine in the liver under dietary protein deficiency and acetaminophen toxic damage. It seems important to reveal the role of interrelated metabolic transformations of sulfur-containing amino acids in the development of nutritional hepatopathologies as well as possible ways of their correction.

\section{Author Contributions}

Conceptualization, H.K.; formal analysis, H.K.; data collection, I.N; writing - original draft preparation, H.K. and I.N; writing - review and editing, H.K. and I.N; supervision, H.K. All authors have read and agreed to the published version of the manuscript.

\section{Funding}

This research received no external funding.

\section{Conflicts of Interest}

The authors declare no conflict of interest.

\section{Bibliography}

1. Batool R., et al. "Protein-energy malnutrition: a risk factor for various ailments". Critical Reviews in Food Science and Nutrition55 (2015): 242-553.

2. Kitada M., et al. "The impact of dietary protein intake on longevity and metabolic health". EBioMedicine 43 (2019): 632640.

3. Kopylchuk HP., et al. "Indexes of citrulline metabolism in rat liver under the toxic injury against the background of alimentary protein deficiency". Ukrainian Biochemical Journal 92 (2020): 113-119.
4. Morris CR., et al. "Acquired Amino Acid Deficiencies: A Focus on Arginine and Glutamine". Nutrition in Clinical Practice 32 (2017): 30-47.

5. Chiew AL., et al. "Interventions for paracetamol (acetaminophen) overdose". Cochrane Database of Systematic Reviews (2018): CD003328.

6. Aminoshariae A and Khan A. "Acetaminophen: old drug, new issues". Journal of Endodontics 41 (2015): 588-593.

7. Normandin PA., et al. "Hidden Danger: Pediatric Acetaminophen Overdose Unintentional and Intentional Emergencies”. Journal of Emergency Nursing 46 (2020): 914-922.

8. Wongm A and Graudins A. "Risk prediction of hepatotoxicity in paracetamol poisoning". Clinical Toxicology (Philadelphia) 55 (2017): 879-892.

9. Ramachandran A and Jaeschke H. "Acetaminophen Hepatotoxicity". Seminars in Liver Disease 39 (2019): 221-234.

10. Popiolek I., et al. "Risk Factors for Hepatotoxicity Due to Paracetamol Overdose in Adults". Medicina (Kaunas) 57 (2021): 752.

11. Shader RI. "Acetaminophen (Paracetamol), COVID-19, and Misleading Conclusions: A Commentary". Journal of Clinical Psychopharmacology 41 (2021): 98-99.

12. Sestili P and Fimognari C. "Paracetamol-Induced Glutathione Consumption: Is There a Link with Severe COVID-19 Illness?" Frontiers in Pharmacology 11 (2020): 579944.

13. Tan SHS., et al. "Medications in COVID-19 patients: summarizing the current literature from an orthopaedic perspective". International Orthopaedics 44 (2020): 1599-1603.

14. Zhou Z., et al. "Orostachys japonicus ameliorates acetaminophen-induced acute liver injury in mice". Journal of Ethnopharmacology 265 (2021): 113392.

15. Torres S., et al. "Endoplasmic Reticulum Stress-Induced Upregulation of STARD1 Promotes Acetaminophen-Induced Acute Liver Failure". Gastroenterology 157 (2019): 552-568.

16. McGill MR and Hinson JA. "The development and hepatotoxicity of acetaminophen: reviewing over a century of progress". Drug Metabolism Reviews 52 (2020): 472-500.

17. Guengerich FP. "Cytochrome P450 2E1 and its roles in disease”. Chemico-Biological Interactions 322 (2020): 109056. 
18. Djuric DM. "Editorial: Sulfur-Containing Amino Acids in Cardiovascular and Neural Physiology, Pathophysiology and Pharmacology: An Overview and Update". Current Medicinal Chemistry 25 (2018): 322-323.

19. Blachier F., et al. "Sulfur-Containing Amino Acids and Lipid Metabolism". Journal of Nutrition 150 (2020): 2524-2531.

20. Karmin $\mathrm{O}$ and Siow YL. "Metabolic Imbalance of Homocysteine and Hydrogen Sulfide in Kidney Disease". Current Medicinal Chemistry 25 (2018): 367-377.

21. Portillo F., et al. "Protein-protein interactions involving enzymes of the mammalian methionine and homocysteine metabolism". Biochimie 173 (2020): 33-47.

22. Škovierová H., et al. "The Molecular and Cellular Effect of Homocysteine Metabolism Imbalance on Human Health". International Journal of Molecular Sciences 17 (2016): 1733.

23. Qureshi SS., et al. "A novel approach in the management of hyperhomocysteinemia". Medical Hypotheses 129 (2019): 109245.

24. Kim J., et al. "Causes of hyperhomocysteinemia and its pathological significance". Archives of Pharmacal Research 41 (2018): 372-383.

25. Kopylchuk HP., et al. "The features of metabolic transformations of homocysteine and cysteine in rats' hepatocytes under the nutritional imbalance. Scientific Herald of Chernivtsi University". Biology (Biological Systems) 12 (2020): 141-149.

26. Saande CJ., et al. "Dietary Egg Protein Prevents Hyperhomocysteinemia via Upregulation of Hepatic Betaine-Homocysteine S-Methyltransferase Activity in Folate-Restricted Rats". Journal of Nutrition 149 (2019): 1369-1376.

27. Pajares MA and Pérez-Sala D. "Mammalian Sulfur Amino Acid Metabolism: A Nexus Between Redox Regulation, Nutrition, Epigenetics, and Detoxification". Antioxidant and Redox Signal 29 (2018): 408-452.

28. Townsend DM., et al. "The importance of glutathione in human disease". Biomedicine and Pharmacotherapy 57 (2003): 145155.

29. Jakubowski H. "Quality control in tRNA charging-editing of homocysteine". Acta Biochimica Polonica 58 (2011): 149-163.

30. Hayes D., et al. "Transport of L-[14C] cystine and L-[14C] cysteine by subtypes of high affinity glutamate transporters over-expressed in HEK cells". Neurochemistry International 46 (2005): 585-594.
31. Randeva HS. "Hormonal Regulation of Homocysteine". Metabolic Syndrome and Related Disorders 1 (2003): 121-128.

32. Adinolfi LE., et al. "Hyperhomocysteinemia and the MTHFR C677T polymorphism promote steatosis and fibrosis in chronic hepatitis C patients". Hepatology 41 (2005): 995-1003.

33. Bublil EM and Majtan T. "Classical homocystinuria: From cystathionine beta-synthase deficiency to novel enzyme therapies". Biochimie 173 (2020): 48-56.

34. Chen H., et al. PLoS One 8 (2013): e76900.

35. Wang M., et al. "The effect of certain conditions in the regulation of cystathionine $\gamma$-lyase by exogenous hydrogen sulfide in mammalian cells". Biochemical Genetics 51 (2013): 503-513.

36. Carsten AW. "Hydrogen sulfide a new gaseous signal molecule and blood pressure regulator". Journal of Nephrology 22 (2009): 173-175.

37. Reeves PG., et al. "AIN-93 purified diets for laboratory rodents: final report of the American Institute of Nutrition ad hoc writing committee on the reformulation of the AIN-76A rodent diet". Journal of Nutrition 123.11 (1993): 1939-1951.

38. Kravchenko L., et al. "A simple non-enzymatic method for the isolation of high yields of functional rat hepatocytes". Cell Biology International 26 (2002): 1003-1006.

39. Zaichko N., et al. "Influence of acute methionine hyperhomocysteinemia on hydrogen sulfide formation in organs of rats and its correction by the complex of vitamins B6, B9, B12. Eksperymentalna ta klinichna fiziolohiia i biokhimiia 4 (2009): 29-35.

40. Murphy ME and Kehrer JP. "Oxidation State of Tissue Thiol Groups and Content of Protein Carbonyl Groups in Chickens with Inherited Muscular Dystrophy". Biochemical Journal 260 (1998): 359-364.

\section{Assets from publication with us}

- Prompt Acknowledgement after receiving the article

- Thorough Double blinded peer review

- Rapid Publication

- Issue of Publication Certificate

- High visibility of your Published work

Website: www.actascientific.com/

Submit Article: www.actascientific.com/submission.php

Email us: editor@actascientific.com

Contact us: +919182824667 